\title{
Perceptual asymmetries are preserved in short-term memory tasks
}

\author{
Leilla Montaser-Kouhsari and Marisa Carrasco \\ New York University, New York, New York
}

\begin{abstract}
Visual performance is heterogeneous at isoeccentric locations; it is better on the horizontal than on the vertical meridian and worse at the upper than at the lower region of the vertical meridian (Carrasco, Talgar, \& Cameron, 2001; Talgar \& Carrasco, 2002). It is unknown whether these performance inhomogeneities are also present in spatial frequency tasks and whether asymmetries present during encoding of visual information also emerge in visual short-term memory (VSTM) tasks. Here, we investigated the similarity of the perceptual and VSTM tasks in spatial frequency discrimination (Experiments 1 and 2) and perceived spatial frequency (Experiments 3 and 4). We found that (1) performance in both simultaneous (perceptual) and delayed (VSTM) spatial frequency discrimination tasks varies as a function of location; it is better along the horizontal than along the vertical meridian; and (2) perceived spatial frequency in both tasks is higher along the horizontal than along the vertical meridian. These results suggest that perceived spatial frequency may mediate performance differences in VSTM tasks across the visual field, implying that the quality with which we encode information affects VSTM.
\end{abstract}

Human visual performance varies across the visual field. Performance decreases with eccentricity in a variety of tasks (e.g., Berkley, Kitterle, \& Watkins, 1975; Carrasco \& Chang, 1995; Carrasco, Evert, Chang, \& Katz, $1995)$ and varies even at isoeccentric locations, causing a horizontal-vertical anisotropy (HVA) and a vertical meridian asymmetry (VMA). Letter recognition as well as orientation discrimination, detection, and localization tasks are better on the horizontal than on the vertical meridian, reflecting HVA. The HVA emerges regardless of stimulus orientation; it is present when the orientation of Gabor stimuli is close to vertical or horizontal - that is, parallel or orthogonal to the meridians (Cameron, Tai, \& Carrasco, 2002; Carrasco, Talgar, \& Cameron, 2001; Mackeben, 1999; Rovamo \& Virsu, 1979; Rovamo, Virsu, Laurinen, \& Hyvärinen, 1982). Contrast sensitivity for all spatial frequencies also decreases more rapidly in the vertical than in the horizontal meridian (Rijsdijk, Kroon, $\&$ van der Wildt, 1980). In addition, performance is superior in the lower than in the upper visual field (Edgar \& Smith, 1990; He, Cavanagh, \& Intriligator, 1996; Levine \& McAnany, 2005; McAnany \& Levine, 2007; Rubin, Nakayama, \& Shapley, 1996). Specifically, it is better on the lower than on the upper region of the vertical meridian, revealing VMA in a variety of tasks such as orientation discrimination, texture segmentation, and Landolt-square acuity tasks (Cameron et al., 2002; Carrasco, Giordano, \& McElree, 2004; Carrasco et al., 2001; Carrasco, Williams, \& Yeshurun, 2002; Fuller, Rodriguez, \& Carrasco, 2008; Talgar \& Carrasco, 2002).

The aims of this study were twofold. First, we wanted to investigate whether these performance inhomogene- ities also emerge in spatial frequency discrimination, by which performance in many spatial resolution tasks is assessed. The findings that HVA and VMA emerge in a Landolt-square acuity task (Carrasco et al., 2002) and a texture segmentation task mediated by spatial resolution (Talgar \& Carrasco, 2002), and that they increase with spatial frequency in orientation discrimination tasks (Cameron et al., 2002; Carrasco et al., 2001; Liu, Heeger, $\&$ Carrasco, 2006), motivated us to seek such inhomogeneities in a task that directly measures spatial frequency discrimination.

Second, we wanted to investigate whether asymmetries present during encoding of visual information also emerge in visual short-term memory (VSTM) tasks. The role of VSTM is to maintain the representation of a visual stimulus over time even after it is no longer present. It is generally accepted that when an observer is asked to compare two sequentially presented stimuli, four different stages are involved: (1) encoding the visual features of the first stimulus, (2) maintaining the representation of the stimulus during the delay, (3) encoding the second stimulus, and (4) comparing the two representations (Regan, 1985). Performance in a VSTM task relies on these stages - specifically, on the encoding stage. Studies using this type of delayed-discrimination tasks have shown that some perceptual attributes of a visual stimulus, such as spatial frequency, orientation, and speed of motion, can be stored for several seconds with little loss of precision if encoded properly (Magnussen, 2000; Magnussen \& Greenlee, 1992; Magnussen, Greenlee, Asplund, \& Dyrnes, 1991; Magnussen, Greenlee, \& Thomas, 1996; Magnussen, Idås, \& Myhre, 1998; Vogels \& Orban,

L. Montaser-Kouhsari, Imk306@nyu.edu 
1986). However, memory of some other attributes such as spatial offset (vernier stimuli), contrast, and direction of motion seem to be retained less robustly (Bisley \& Pasternak, 2000; Fahle \& Harris, 1992; Lee \& Harris, 1996; Nilsson \& Nelson, 1981; Pasternak \& Greenlee, 2005; Vogels \& Orban, 1986).

Visual information cannot be retrieved from short-term memory properly if it is not encoded or stored accurately. For instance, in a delayed-discrimination task, the presence of a mask during the delay period interferes with task performance (Lalonde \& Chaudhuri, 2002; Pasternak \& Greenlee, 2005; Pasternak \& Zaksas, 2003). This phenomenon, known as "memory masking" (Magnussen et al., 1991), occurs if the mask shares a similar property with the remembered stimulus but is distinctly different. The selective interference of the mask illustrates that information of the visual attributes, such as spatial frequency, is preserved in memory by mechanisms closely related to those involved in encoding (Pasternak \& Greenlee, 2005). Therefore, if masking causes deterioration of performance in VSTM tasks, any other factor that also affects representation of information could influence performance in VSTM tasks.

In the first part of this study, we investigated whether performance varies as a function of stimulus location in (1) a simultaneous spatial frequency discrimination task and (2) a delayed spatial frequency discrimination task (VSTM). In particular, we explored whether each of these tasks yields HVA and VMA. In the second part of this study, we examined whether perceived spatial frequency, assessed by the point of subjective equality (PSE), changes as a function of stimulus location in (1) a simultaneous spatial frequency discrimination task and (2) a delayed spatial frequency discrimination task. We hypothesized that if simultaneous spatial frequency discrimination and perceived spatial frequency varied across cardinal locations, these differences would affect performance in the corresponding delayed tasks, resulting in heterogeneous performance and perceived spatial frequency across cardinal locations. Alternatively, the performance and perceived differences in the simultaneous tasks could become either more pronounced or attenuated in the delayed tasks.

In Experiment 1, we investigated whether simultaneous spatial frequency discrimination varies as a function of stimulus location, resulting in performance inhomogeneities at isoeccentric locations. In Experiment 2, we examined whether such inhomogeneities would also emerge in a VSTM task. To do so, we measured performance in a two-interval forced choice (2IFC) delayed spatial frequency discrimination task.

\section{EXPERIMENT 1}

\section{Method}

Participants. Seven observers, 25- to 30-year-old graduate students with normal or corrected-to-normal vision, participated voluntarily in the experiment. All observers were psychophysically trained but naive with respect to the purpose of the experiment. The experimental protocol was approved by the New York University committee on activities involving human participants.
Apparatus. Stimuli for the experiment were generated using MATLAB and Psychophysics Toolbox extensions (Brainard, 1997; Pelli, 1997) with an Apple G4 Power Macintosh computer and were displayed on a gamma-corrected CTX PR 1400 21-in. monitor $(1,024 \times 768$ pixels; $75 \mathrm{~Hz})$. A video attenuator drove only the green gun, to increase the possible set of distinct luminance levels (12 bits). The observers were placed in a dark room, instructed to fixate, and viewed the stimuli binocularly from a distance of $57 \mathrm{~cm}$, with their heads held in place by chin- and forehead rests.

Stimuli. The stimulus sequence consisted of a fixation display and a Gabor display. The fixation display contained a black fixation circle with the diameter of $0.2^{\circ}$ at the center of a green background $\left(17.5 \mathrm{~cd} / \mathrm{m}^{2}\right)$ and two placeholder frames determining where the two stimuli would be presented. The Gabor display contained the fixation circle, the two placeholder frames, and two $100 \%$ contrast vertical Gabor patches (sinusoidal gratings enveloped in a Gaussian window; $S D, 0.3^{\circ}$ ) at $6^{\circ}$ eccentricity. On each trial, two Gabors, the standard and test, appeared simultaneously at any two of four possible locations: East (E), West (W), North (N), and South (S). The standard stimulus had a spatial frequency of 6 cycles per degree (cpd), and the test stimulus had a spatial frequency that differed from the standard by a fixed amount and was either higher or lower than the standard. The direction of the difference between test and standard was chosen randomly on each trial. The spatial frequency difference between test and standard was adjusted for each observer individually to an approximate constant overall level ( $75 \%)$, yielding performance above chance and below perfect performance at all locations. The range of differences between the standard's and test's spatial frequency across observers was $0.4-0.6 \mathrm{cpd}$. The ISI duration, as well as the order and location of the test and standard stimuli, was chosen randomly on each trial.

Procedure. Each observer completed 50 practice trials and 350 experimental trials. Each trial consisted of a $200-\mathrm{msec}$ fixation display followed by a 100 -msec stimulus presentation, so that eye movements were precluded while the display was on (Deubel \& Schneider, 1996; Hoffman \& Subramaniam, 1995; Mayfrank, Kimmig, \& Fischer, 1987). Observers performed a two-alternative forced choice (2AFC) task. They were asked to report which location (N, E, S, or $\mathrm{W})$ contained the stimulus with the higher spatial frequency by pressing one of four keys corresponding to those locations. The key locations matched the location of the stimulus on the screen. Observers had $2 \mathrm{sec}$ to respond, and they received auditory feedback for incorrect responses (see Figure 1A).

\section{Results}

Accuracy (proportion correct) of the spatial frequency discrimination task varied as a function of location; it was better along the horizontal than along the vertical meridian, revealing an HVA (Figure 2A). Accuracies were averaged across observers for four conditions: N\&E/N\&W, $\mathrm{N} \& \mathrm{~S}, \mathrm{E} \& \mathrm{~W}$, and $\mathrm{S} \& \mathrm{E} / \mathrm{S} \& \mathrm{~W}$. The letters correspond to the locations of the two stimuli (test and standard); for example, N\&S means that one stimulus was presented at the $\mathrm{N}$ location and the other at the $\mathrm{S}$ location. $\mathrm{N} \& \mathrm{E} / \mathrm{N} \& \mathrm{~W}$ is the average of the mean accuracies in N\&E and N\&W. A repeated measures ANOVA performed on the above conditions $(\mathrm{N} \& \mathrm{E} / \mathrm{N} \& \mathrm{~W}, \mathrm{~N} \& \mathrm{~S}, \mathrm{E} \& \mathrm{~W}$, and $\mathrm{S} \& \mathrm{E} / \mathrm{S} \& \mathrm{~W})$ revealed a significant main effect of location $[F(3,18)=$ $4.15, p<.05]$. Performance was significantly better when the test and the standard stimuli were presented along the horizontal meridian $(\mathrm{E} \& \mathrm{~W})$ rather than along the vertical meridian (N\&S) $[t(6)=3.45, p=.01]$ or when either stimulus appeared at the $\mathrm{N}[t(6)=2.62, p<.05]$ or at the $\mathrm{S}[t(6)=3.96, p<.01]$ location. (All $t$ tests were two-tailed.) 
A

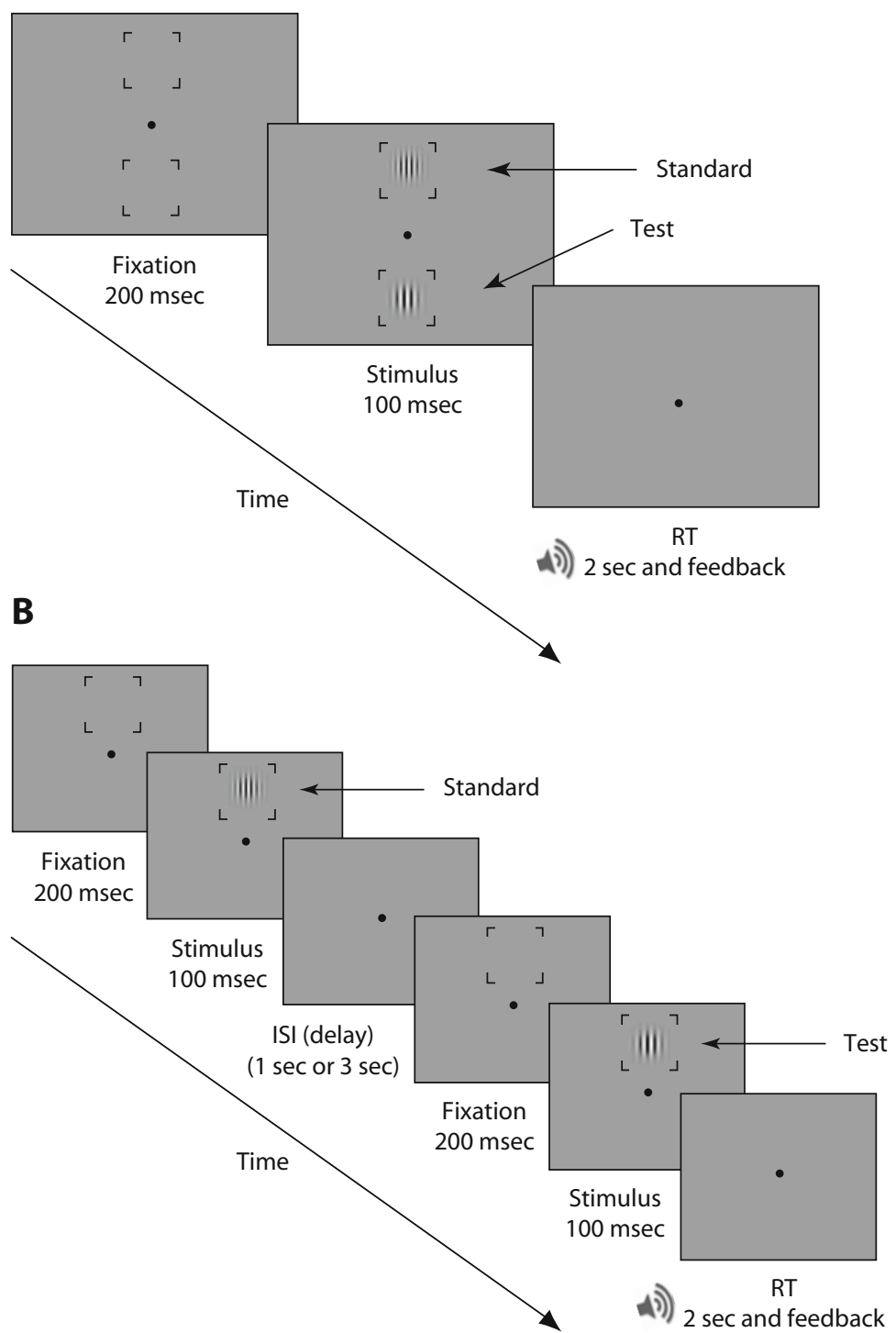

Figure 1. Procedure. (A) Experiment 1. In each trial, two stimuli (test and standard) were presented simultaneously. Observers were asked to report which stimulus had a higher spatial frequency. (B) Experiment 2. Each trial consisted of two intervals separated by a delay. In each interval a Gabor stimulus (test or standard) was presented at a peripheral location (North, South, East, or West). Observers were asked to report whether the stimulus in the first or second interval had a higher spatial frequency. (This figure is not representative of the actual stimuli in terms of eccentricity and spatial frequency). ISI, interstimulus interval; RT, response time.

\section{EXPERIMENT 2}

\section{Method}

Participants. Eight observers, 25- to 30-year-old graduate students with normal or corrected-to-normal vision, participated voluntarily in the present experiment. Five of them had also participated in Experiment 1. All were naive with respect to the purpose of the experiment.

Apparatus and Stimuli. The apparatus and the stimuli were the same as those in Experiment 1.The stimulus sequence consisted of (1) a fixation display containing a black fixation circle with a diam- eter of $0.2^{\circ}$ at the center of a green background $\left(17.5 \mathrm{~cd} / \mathrm{m}^{2}\right)$ and a placeholder frame at which the stimulus was to be presented; (2) the first stimulus display (first interval); (3) an interstimulus interval (ISI; 1 or $3 \mathrm{sec}$ ); (4) the second fixation display, consisting of the fixation circle and the placeholder frame; and (5) the second stimulus display (second interval). Each stimulus display contained a single Gabor patch and a frameholder. The stimuli in both intervals were centered at $6^{\circ}$ eccentricity and both appeared at the same location of the screen-W, E, N, or S-for $100 \mathrm{msec}$ (Figure 1B). In all other respects, the stimuli were similar to those of Experiment 1, except that the standard had a spatial frequency of $7 \mathrm{cpd}$. 


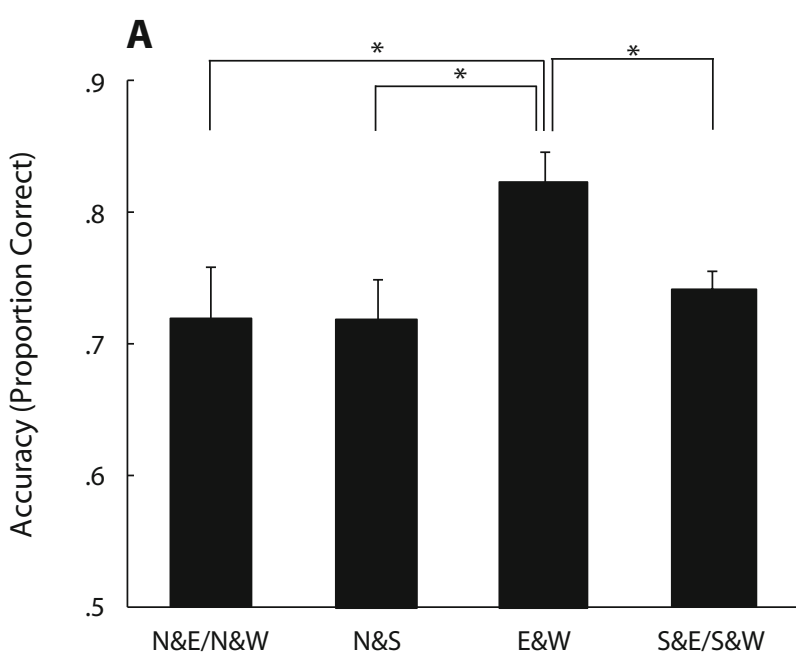

Location of the Test and Standard Stimuli

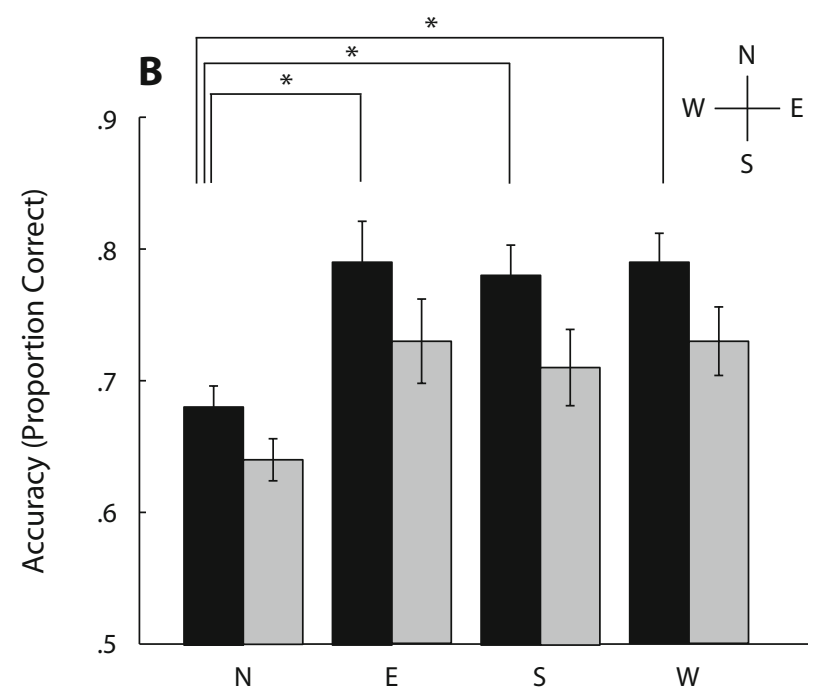

Location of the Standard and Test Stimuli

Figure 2. Results for spatial frequency discrimination tasks. (A) Experiment 1. Accuracy (proportion correct) averaged across observers for four conditions. The letters correspond to the locations of the two stimuli: North (N), South (S), East (E), and West (W). N\&S, one stimulus was presented at the $N$ and the other at the $S$ location; $N \& E / N \& W$, average of accuracies in $N \& E$ and $N \& W$ conditions. (B) Experiment 2. Accuracy across observers for four conditions when both the test and the standard appeared at each peripheral location. Black and gray bars show the accuracy for the 1-sec and 3-sec delay, respectively. Asterisks indicate that performance was significantly different $\left({ }^{*} p<.05\right)$. Error bars represent standard error of the mean across the observers, calculated using the method of Loftus and Masson (1994).

Procedure. Observers completed 50 practice trials and 800 experimental trials. Each trial consisted of two intervals separated by an ISI (delay) that lasted either 1 or $3 \mathrm{sec}$. Observers performed a 2IFC task and reported which interval (first or second) contained the stimulus with the higher spatial frequency by pressing Key 1 or Key 2. Observers had $2 \mathrm{sec}$ to respond and received auditory feedback for incorrect responses.

\section{Results}

Accuracy (proportion correct) in the delayed spatial frequency discrimination task varied as a function of location and delay (see Figure 2B). Performance did not differ with regard to the order of the standard and test. A 4 (location: N, E, S, and W) $\times 2$ (delay: 1 and 3 ) repeated measures ANOVA revealed significant main effects of delay $[F(1,7)=50.33, p<.001]$, with overall performance higher for the 1-sec than for the 3-sec delay, and of location $[F(3,21)=6.56, p<.005]$, but no significant two-way interaction $(F<1)$. To explore the effect of location, a post hoc paired $t$ test showed a lower accuracy when both the test and the standard were shown at $\mathrm{N}$ than when they were shown at $\mathrm{E}[t(7)=3.14, p<.05$ and 4.12, $p<.005], \mathrm{W}[t(7)=4.42, p<.005$ and 4.11, $p<$ $.005]$, or $\mathrm{S}[t(7)=7.13, p<.001$ and 2.24, $p=.06]$ for the 1-sec and 3-sec delays, respectively. Taken together, these results showed that performance in VSTM task also suffered along the vertical meridian, specifically at the $\mathrm{N}$ location, and suggest that performance differences in the spatial frequency discrimination tasks (Experiment 1) carried over to this VSTM delayed spatial frequency task.

The finding that performance in spatial frequency discrimination is better along the horizontal than along the vertical meridian (Experiment 1) could be related to stimulus appearance. For example, it is possible that the apparent spatial frequency of a stimulus differs across the meridians and that this difference affects task performance. It has been established that both perceived contrast (Fuller et al., 2008) and performance on tasks that assess contrast sensitivity (Cameron et al., 2002; Carrasco et al., 2001) differ across locations. In Experiment 3, we tested the hypothesis that perceived spatial frequency could also vary across meridians, by keeping the location of the standard fixed (at the center of the screen) and varying the location of the test (N, E, W, or S).

Furthermore, in Experiment 4, we investigated whether a spatial frequency discrimination difference is present in a VSTM task by using a delayed spatial frequency discrimination task. Given that the PSEs are similar over time in delayed contrast and contour curvature discrimination tasks (Lee \& Harris, 1996; Sakai, 2003), we predicted that if there were differences in perceived spatial frequency across the meridians, they would transfer over time and a similar pattern of PSEs would be obtained in the VSTM task.

\section{EXPERIMENT 3}

\section{Method}

Participants. Five observers, 25- to 30-year-old graduate students with normal or corrected-to-normal vision, participated voluntarily in the experiment. Three of these observers had participated in both Experiments 1 and 2, and 1 observer had participated in Experiment 1 . All observers were psychophysically trained but naive with respect to the purpose of the experiment.

Apparatus. The stimuli were generated using MGL programming software (http://justingardner.net/mgl) with an Apple G5 Power Macintosh computer and were displayed on a gammacorrected, Hewlett-Packard P1230 21-in. CRT monitor (1,152 × 870 pixels; $75 \mathrm{~Hz}$ ). On each trial, two Gabor patches were pre- 

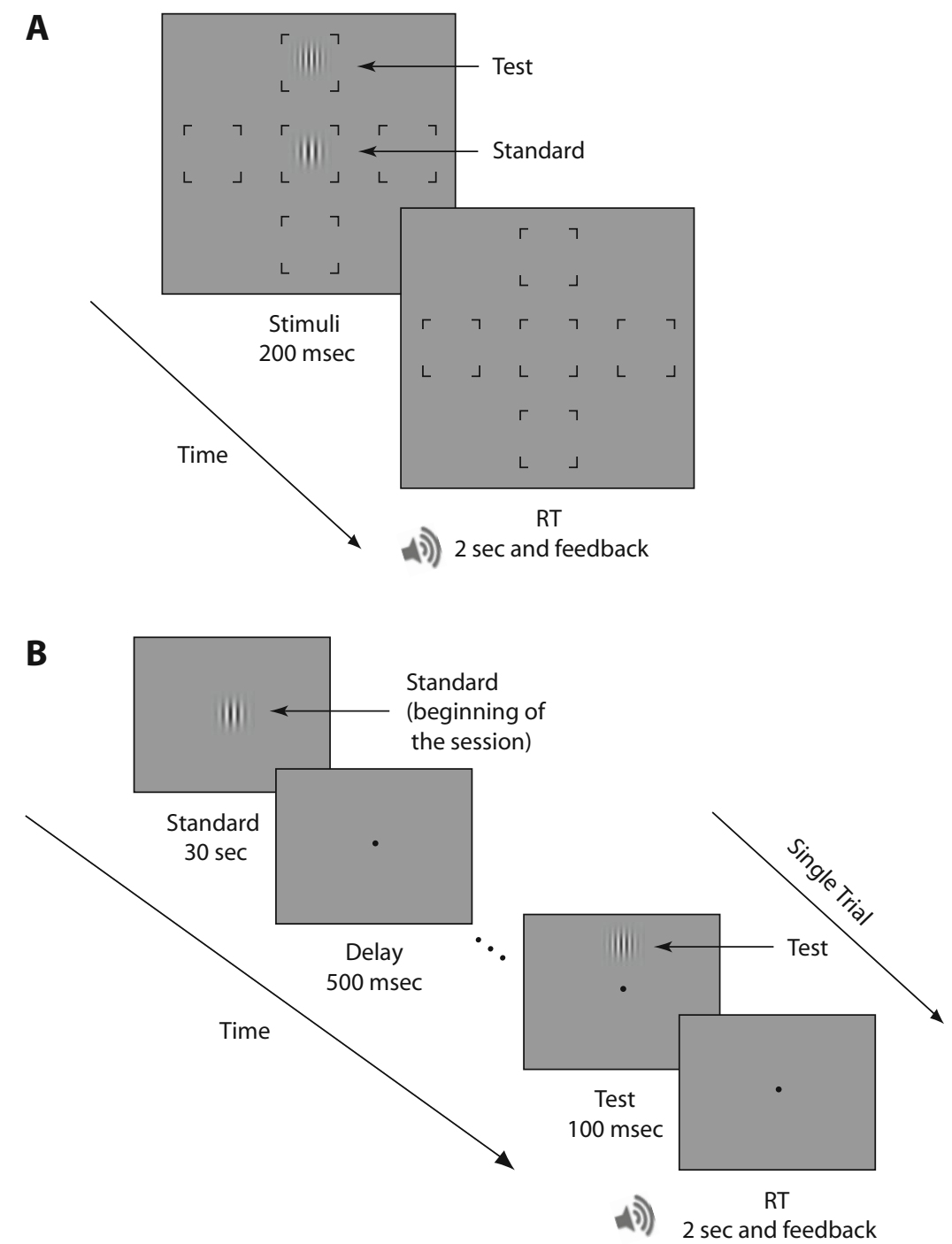

Figure 3. Procedure. (A) Experiment 3. In each trial two stimuli were presented simultaneously; test at a peripheral location, and standard at the center. Observers were asked to report which stimulus had a higher spatial frequency. (B) Experiment 4. Each session started with the presentation of the standard stimulus and continued with the presentation of the test trials at a peripheral location $(\mathrm{N}, \mathrm{S}, \mathrm{E}$, or W). Observers were asked to report which stimulus (the memorized standard or the test) had a higher spatial frequency. (This figure is not representative of the actual stimuli in terms of eccentricity and spatial frequency.) RT, response time.

sented. The size, orientation, and contrast of the Gabor stimuli were the same as in the previous experiments. The display contained five placeholder frames present throughout the trial. The standard stimulus was presented at the center, and the test was presented on the $\mathrm{W}, \mathrm{E}, \mathrm{N}$, or $\mathrm{S}$ of a gray background $\left(40.8 \mathrm{~cd} / \mathrm{m}^{2}\right)$ (Figure $3 \mathrm{~A}$ ). To maintain variation between trials, two different standard stimuli were used. On each trial, the standard's spatial frequency was either 6.5 or $7 \mathrm{cpd}$. When the standard had a spatial frequency of $6.5 \mathrm{cpd}$, the spatial frequency of the test was chosen from the following values: $5.5,6,6.5,7$, and $7.5 \mathrm{cpd}$. When the standard had a spatial frequency of $7 \mathrm{cpd}$, the test spatial frequency was chosen from the values $6,6.5,7,7.5$, and $8 \mathrm{cpd}$. The location of the test stimulus and the spatial frequency and phase of the test and standard stimuli were chosen randomly on each trial.

Procedure. Each observer completed 50 practice trials and 1,000 experimental trials. Each trial consisted of a stimulus-presentation display during which the test and the standard appeared for $200 \mathrm{msec}$ (Figure 3A). The timing of the stimulus-presentation precluded goal-directed eye movements (Deubel \& Schneider, 1996; Hoffman \& Subramaniam, 1995; Mayfrank et al., 1987). Observers performed a $2 \mathrm{AFC}$ task reporting which stimulus had a higher spatial frequency by pressing Key 1 (central) or Key 2 (peripheral). They had $2 \mathrm{sec}$ to respond and received auditory feedback for incorrect responses.

\section{Results}

Perceived spatial frequency varied as a function of location. Figure 4A shows the four group-averaged psychometric functions averaged across the two standard stimuli. The functions show the proportion of trials on which the observers chose the test Gabor as the stimulus with higher 


\section{A Simultaneous (Experiment 3)}
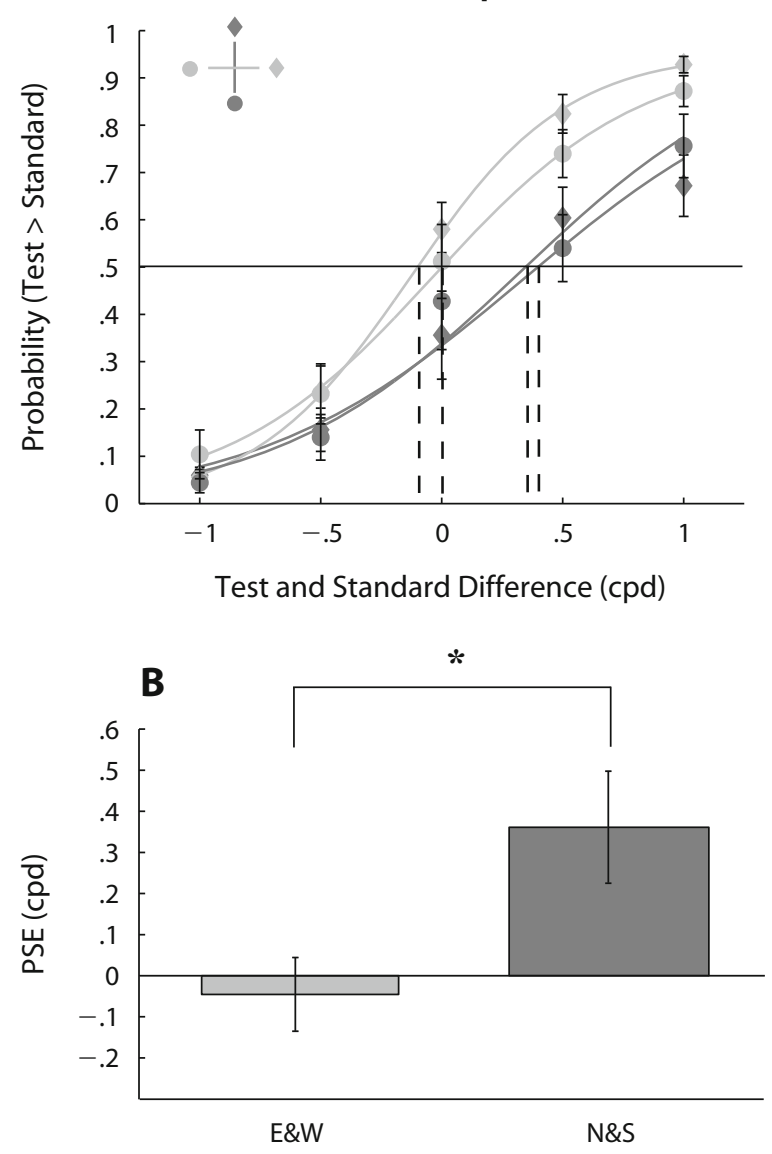

Location of the Test Stimulus

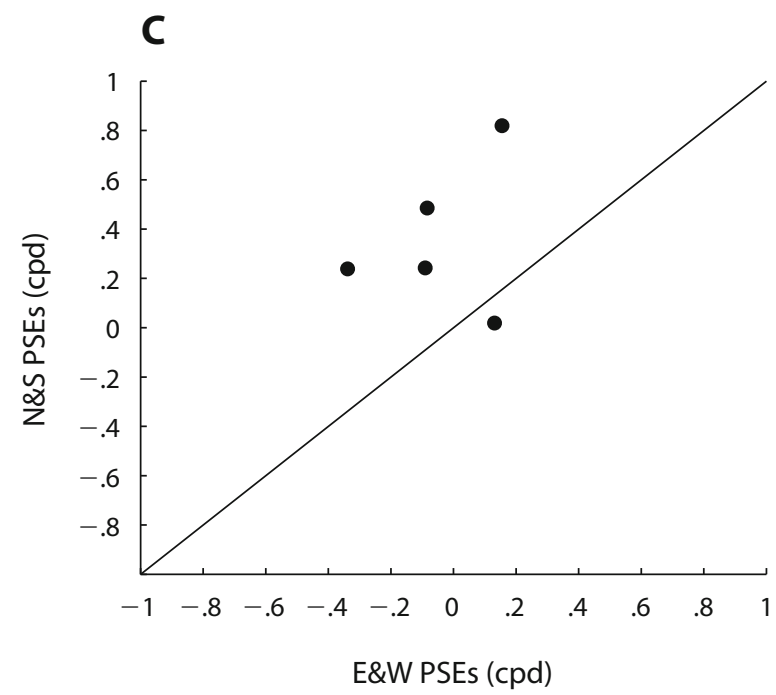

D

VSTM (Experiment 4)
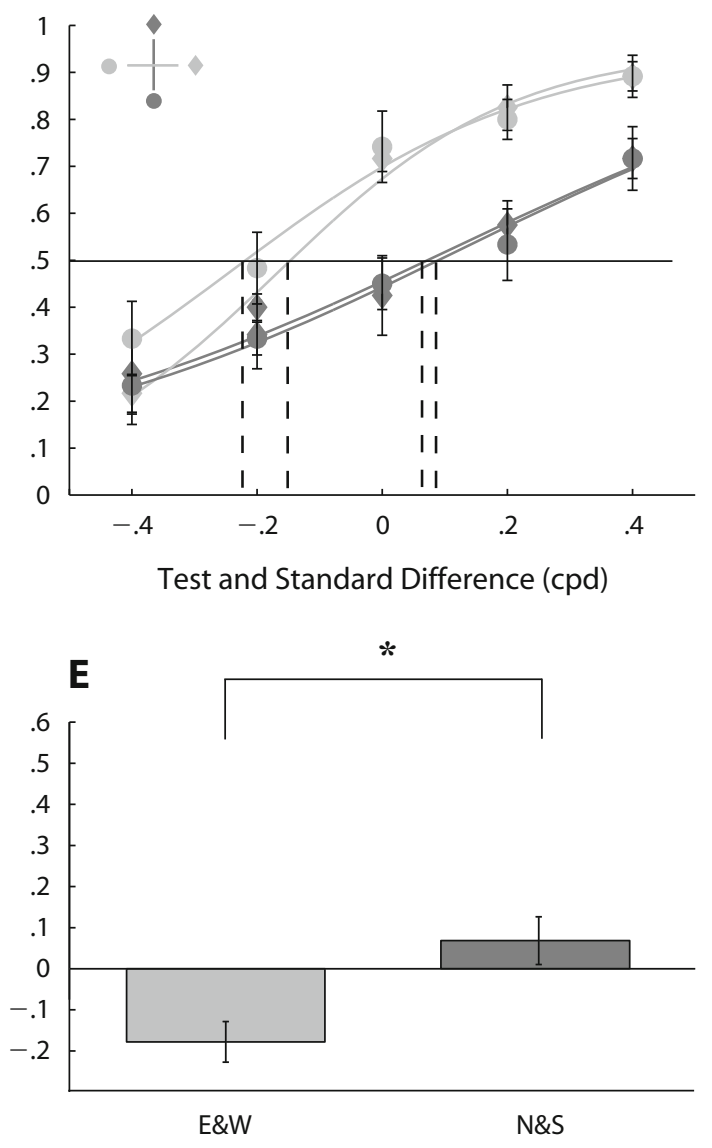

Location of the Test Stimulus

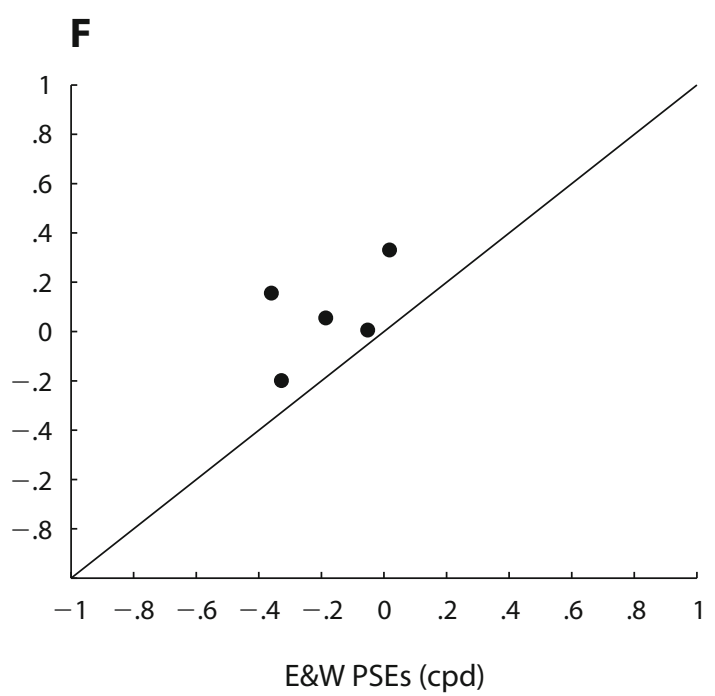

Figure 4. Result for perceived spatial frequency tasks. Top row: Psychometric functions for the simultaneous task (A, in Experiment 3) and delayed (VSTM) task (D, in Experiment 4). Each function shows proportion of trials on which observers chose the test Gabor as having a higher spatial frequency than the standard Gabor. The horizontal line at .5 proportion correct shows chance performance, and the vertical dashed lines indicate for each location the point of subjective equality (PSE), at which the test and standard were equally likely to be reported as having higher spatial frequency. Middle row: Averaged point of subjective equality (PSE) along the horizontal meridian (E\&W) and the vertical meridian (N\&S) for Experiment 3 (B), and Experiment 4 (E). Bottom row: Scatterplots of individual observers' PSEs when the test stimulus was presented along the vertical meridian (N\&S) versus the horizontal meridian $(E \& W)$ for Experiment $3(C)$ and Experiment $4(F)$. Asterisks indicate a statistically significant difference $(* p<.05)$. Error bars represent the standard error of the mean across the observers. 
spatial frequency. Each psychometric function was fit with a logistic function. Fits were attained using maximum likelihood estimation, and goodness of fit was evaluated using deviance scores calculated as the log-likelihood ratio between the fully saturated, zero residual model and the data model (Wichmann \& Hill, 2001a, 2001b) (http:// bootstrap-software.org/psignifit). All scores (except for 1 out of 20 fits) were below the critical chi-square value $\left[\chi^{2}(5)<11.07, \mathrm{CI}=0.95\right]$, indicating good fits. The stimulus appeared to have lower spatial frequency when it was located along the vertical meridian than when it was located along the horizontal meridian. There was a rightward shift in PSEs when the test stimulus was presented at the $\mathrm{N}$ and $\mathrm{S}$ rather than at the $\mathrm{W}$ and $\mathrm{E}$.

To compute the averaged PSEs (test spatial frequency at which the observer was equally likely to report the test or the standard as having higher spatial frequency), we fit the data for each of the four locations separately, which yielded four psychometric functions per observer. PSEs were averaged for the vertical (N\&S) and horizontal (E\&W) meridians across the 5 observers. The PSEs did not differ significantly either between $\mathrm{N}$ and $\mathrm{S}$ or between $\mathrm{E}$ and W. Figure 4B shows averaged PSEs across all observers along the vertical $(\mathrm{N} \& S)$ and the horizontal $(\mathrm{E} \& \mathrm{~W})$ meridians. Perceived spatial frequency was lower at the vertical than at the horizontal meridian $[t(4)=2.89, p<$ $.05]$ : Observers needed higher spatial frequency $(0.4 \mathrm{cpd})$ along the former than along the latter to reach the point of subjective equality. The effect of location on perceived spatial frequency was highly consistent across observers. Figure 4C shows the individual PSEs in the perceptual task. All but one of the PSEs fell above the unity line, indicating that they were higher when the test was located along the vertical than along the horizontal meridian.

Spatial frequency discrimination accuracy (proportion correct) also varied as a function of location and spatial frequency. A repeated measures ANOVA showed marginal main effects of location of the test stimulus $[F(1,4)=$ $5.97, p<.1]$ and of spatial frequency $[F(1,4)=7.7, p=$ $.05]$, but there was a significant two-way interaction between these factors $[F(1,4)=8.06, p<.05]$ : Accuracy was significantly higher when the test appeared along the horizontal meridian than when it appeared along the vertical meridian, and its spatial frequency was higher than that of the standard $[t(4)=3.33, p<.05]$ but not when it was lower $(p>.1$; Figure $5 \mathrm{~A})$. This pattern was consistent for all observers. Figure 5B shows the individual accuracy differences when the test was located along the horizontal and the vertical meridians. All but one of the difference values were greater when the spatial frequency of the test was higher than the standard (points fall below the unity line).

\section{EXPERIMENT 4}

\section{Method}

Participants. Five observers, 25- to 30-year-old graduate students with normal or corrected-to-normal vision, participated voluntarily in the experiment. All of them were trained psychophysical observers but were naive with respect to the purpose of the experiment. All of the observers participated in at least two out of the three previous experiments (except 1 who did not participate in Experiment 2).

Apparatus. The apparatus was the same as in Experiment 2.

Stimuli. Both standard and test stimuli were Gabor patches (the same as in the three previous experiments). The test stimulus was presented at $6^{\circ}$ eccentricity on a green background $\left(17.5 \mathrm{~cd} / \mathrm{m}^{2}\right)$. The standard stimulus had a spatial frequency of $7 \mathrm{cpd}$ and was located at the center of the display. The test stimulus had one of five spatial frequencies $(6.6,6.8,7,7.2$, and $7.4 \mathrm{cpd})$. The spatial frequency and the location of the test Gabor were chosen randomly on each trial.

Procedure. Each observer completed 50 practice trials and 480 experimental trials. The experimental session started with the presentation of the standard stimulus for $30 \mathrm{sec}$ (to ensure that observers could encode and remember its spatial frequency throughout the session), followed by $500 \mathrm{msec}$ of fixation, and continued with the presentation of the test trials. A trial consisted of a $100-\mathrm{msec}$ test stimulus at a peripheral location (N, E, W, or S; Figure 3B). Observers were asked to report which Gabor (the test or the standard) had a higher spatial frequency by pressing Key 1 or Key 2. They had a maximum of $2 \mathrm{sec}$ to respond and received auditory feedback for incorrect responses. Each session consisted of four blocks of 120 trials, each lasting a maximum of $4 \mathrm{~min}$.

\section{Results}

The comparison of the test spatial frequency to the memorized standard spatial frequency varied as a function of location. Figure 4D shows the four group-averaged psychometric functions estimated from the data, corresponding to the location of the test stimulus (as in Experiment 3). All 20 deviance scores were below the critical chi-square value $\left[\chi^{2}(5)<11.07, \mathrm{CI}=0.95\right]$, indicating good fits.

PSEs were computed as described in Experiment 3. Figure 4E shows averaged PSEs across all observers for the vertical meridian (N\&S) compared with the horizontal meridian (E\&W). Results showed that PSEs in the delayed spatial frequency discrimination varied as a function of location. The PSEs were $0.3 \mathrm{cpd}$ higher when the test stimulus was presented along the vertical meridian than when it was presented along the horizontal meridian $[t(4)=4.67$, $p<.05]$. This showed that the test stimulus was reported to have higher spatial frequency than the memorized standard when it was located along the horizontal rather than along the vertical meridian. This difference between PSEs is consistent with the difference found in the spatial frequency discrimination task (Experiment 3). Figure 4F shows that the effect of location on perceived spatial frequency was highly consistent across observers; the individual PSEs in the VSTM task were higher when the test was located along the vertical meridian than when it was located along the horizontal meridian (points fall above the unity line). In addition, accuracy (proportion correct) in the delayed spatial frequency discrimination task also varied as a function of location and spatial frequency. A repeated measures ANOVA revealed no significant main effects of location $(p=.1)$ or spatial frequency $(p>.1)$, but a significant two-way interaction between these two factors $[F(1,4)=10.64, p<.05]$. Accuracy was significantly higher when the test appeared along the horizontal meridian than when it appeared along the vertical meridian, and its spatial frequency was higher than that of the memorized standard $[t(4)=8.60, p<.001]$ but not when its spatial frequency was lower $(p>.1$; Figure 5C). Fig- 


\section{A Simultaneous (Experiment 3)}

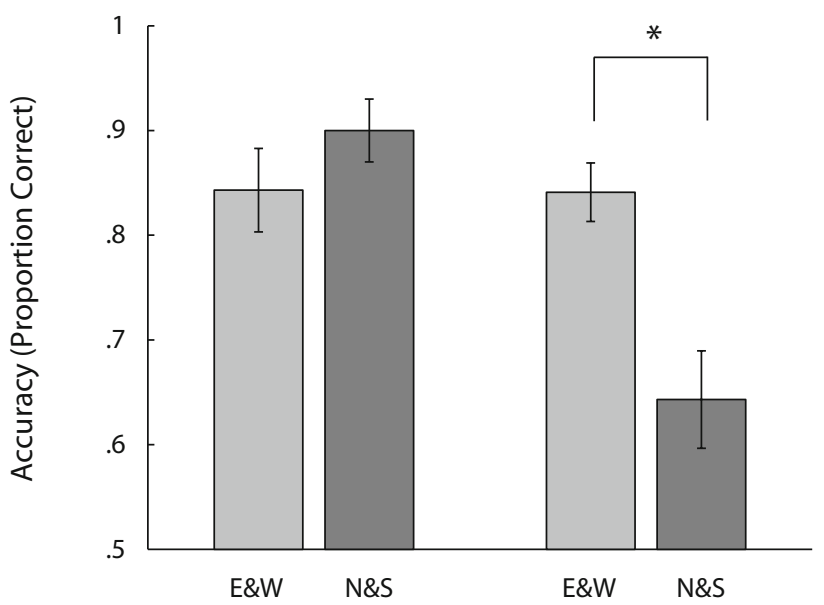

Test SF $<$ Standard SF Test SF $>$ Standard SF

B

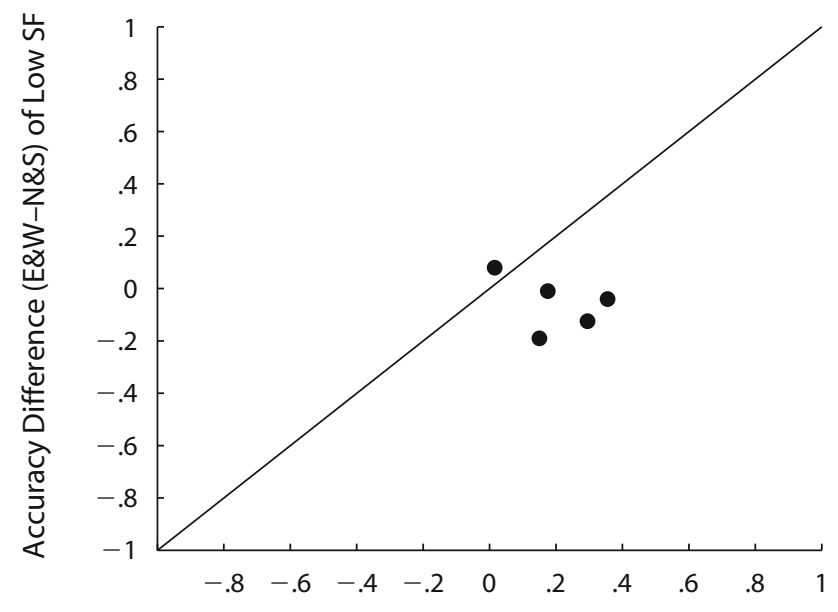

Accuracy Difference (E\&W - N\&S) of High SF
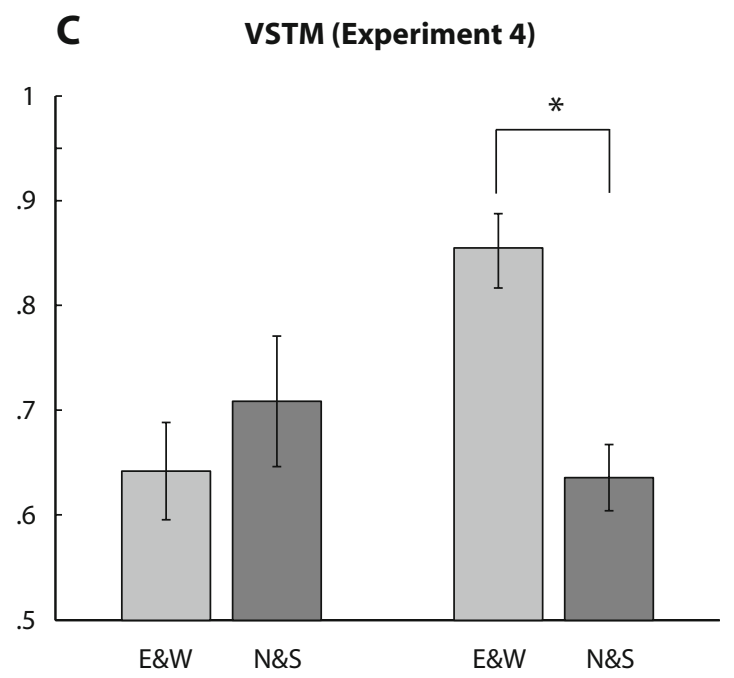

Test SF $<$ Standard SF Test SF $>$ Standard SF
D

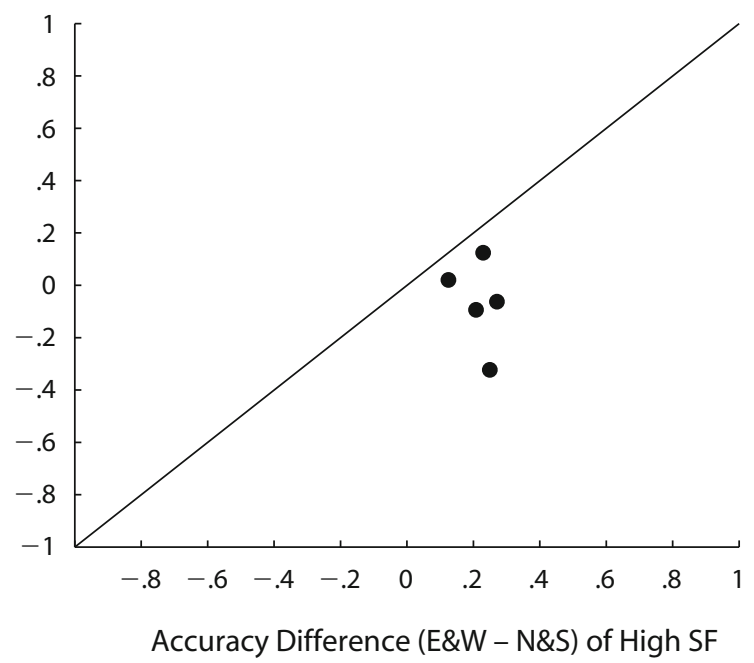

Figure 5. Top row: Spatial frequency (SF) discrimination performance (proportion correct) when the spatial frequency of the test was lower or higher than the standard along the horizontal (E\&W) and vertical (N\&S) meridians for Experiment 3 (A) and Experiment 4 (C). Bottom row: Scatterplots of individual observers show the accuracy difference (proportion correct) between horizontal and vertical meridians when the spatial frequency of the test was lower than the standard versus when it was higher than the standard, for Experiment 3 (B) and Experiment 4 (D). Asterisks indicate a statistically significant difference $(* p<.05)$. Error bars represent the standard error of the mean across the observers.

ure 5D shows the individual accuracy differences when the test was located along the horizontal and vertical meridians. All the difference values were greater when the spatial frequency of the test was higher than that of the memorized standard (points fell below the unity line).

\section{DISCUSSION}

The present results show that location affects processing of spatial frequency in discrimination and VSTM tasks. Spatial frequency discrimination is better along the horizontal than along the vertical meridian (Experiment 1). VSTM performance follows the same pattern of asymmetry found in the spatial frequency discrimination task (Experiment 2). Correspondingly, perceived spatial frequency differs across the meridians in both the perceptual (Experiment 3) and VSTM (Experiment 4) tasks.

\section{Spatial Frequency Discrimination in Simultaneous and Delayed (VSTM) Tasks}

The present results indicate that performance in a spatial frequency discrimination task varies as a function of location. It is better along the horizontal than along the vertical meridian. This finding of a horizontal vertical asymmetry (HVA) for spatial frequency discrimination is consistent with an HVA for contrast sensitivity (Cam- 
eron et al., 2002; Rijsdijk et al., 1980). We note that previous studies have shown that the shape of the performance fields is independent of stimulus orientation; that is, independent of the alignment of the stimuli with the meridian (Carrasco et al., 2001). Moreover, at far peripheral locations, resolution is better for oriented gratings with parallel as opposed to perpendicular orientation to the meridians (Rovamo et al., 1982). Thus, had the alignment of the stimuli with the vertical meridian affected our results, the opposite results should have been found; that is, better performance along the vertical than along the horizontal meridian.

We also examined VSTM as a function of location. Performance in a delayed spatial frequency discrimination task was better along the horizontal than along the vertical meridian (HVA) and better at the $\mathrm{S}$ than at the $\mathrm{N}$ location (VMA). The analogous pattern of results (HVA) found for the delayed and the simultaneous spatial frequency tasks, as well as for other perceptual tasks (Cameron et al., 2002; Carrasco et al., 2001, 2002, 2004), suggests that parameters that affect performance in perceptual tasks also operate on the encoding processing of VSTM. Therefore, we conclude that low-level factors (retinal and cortical inhomogeneities) that cause the difference in spatial frequency encoding across the meridians could be responsible for the dissimilarity found in the VSTM task performance across the meridians.

Delayed-discrimination studies have revealed that perceptual attributes of a visual stimulus, such as spatial frequency, can be stored for several seconds with little loss of precision (Magnussen, 2000; Magnussen \& Greenlee, 1992, 1999; Magnussen et al., 1991, 1996, 1998; Vogels \& Orban, 1986). However, the present results show a slight but significant decrease in performance of delayed spatial frequency discrimination. Increasing the delay from 1 to $3 \mathrm{sec}$ decreased performance at all locations, but did not alter the inhomogeneities across isoeccentric locations. The slight decrement in performance at all locations is consistent with the hypothesis that the memory representations for discrimination might remain at the encoded value but become noisier with the passage of time. This hypothesis is based on the finding that contrast discrimination thresholds increase with time (Lee \& Harris, 1996).

The inconsistency between our findings and those of previous studies could be due to differences in the methodologies used. For instance, we assessed performance with the method of constant stimuli, whereas previous studies assessed performance at threshold with staircases; we introduced stimulus uncertainty by varying the location of the standard and the test stimulus in each trial, whereas in previous studies both stimuli were presented only foveally (where resolution is higher) at one location (i.e., without uncertainty). Indeed, Cornelissen and Greenlee (2000) have concluded that high-precision VSTM is a foveal specialization. They showed that memory performance declines with eccentricity, approximately as a Gaussian function of location, probably due to the lower resolution and sensitivity of eccentric vision or to the limited capacity of visual attention.

\section{Perceived Spatial Frequency in Simultaneous and Delayed (VSTM) Tasks}

Using a high-contrast stimulus, we found that perceived spatial frequency was higher along the horizontal than along the vertical meridian. PSEs were higher at the $\mathrm{N}$ and $\mathrm{S}$ than at the $\mathrm{W}$ and $\mathrm{E}$ locations, indicating that observers needed a higher spatial frequency along the vertical than along the horizontal meridian to attain the PSE. This finding could be related to the finding that discrimination performance is better for the horizontal than for the vertical meridian. In particular, accuracy was higher along the horizontal than along the vertical meridian when the test stimulus had a higher spatial frequency than the standard stimulus.

Furthermore, we observed that this HVA was only evident when the test stimulus had a higher spatial frequency. These results are consistent with the findings that in orientation discrimination, detection, and localization tasks, the HVA becomes more pronounced with higher spatial frequency (Carrasco et al., 2001). Moreover, the magnitude of the HVA in a Landolt-square acuity task, which measures spatial resolution, increases as gap size decreases (Carrasco et al., 2002).

It has been reported that there is a lower density of cones and ganglion cells in the peripheral retina than in the fovea (Curcio, Sloan, Kalina, \& Hendrickson, 1990; Perry \& Cowey, 1985). Similarly, the density of ganglion cells is lower along the vertical than along the horizontal meridian (Curcio \& Allen, 1990; Perry \& Cowey, 1985). In addition, cone density declines faster with distance from the fovea along the vertical than it does along the horizontal meridian (Curcio et al., 1990). On the basis of these findings, we propose that the HVA in perceived spatial frequency could be due to a lower density of cones and ganglion cells along the vertical meridian.

An HVA also emerged in the VSTM task - that is, location affected perceived spatial frequency. The similar pattern of results for simultaneous and delayed (VSTM) tasks is consistent with psychophysical studies showing similar PSEs over time in delayed contrast and contour curvature discrimination tasks (Lee \& Harris, 1996; Sakai, 2003). The present finding provides support for the conclusion that "perception and memory share common representations in the sense that the fidelity with which an image is coded will determine the quality of the information retrieved from memory" (Magnussen \& Greenlee, 1999, p. 90).

\section{Perceived Spatial Frequency and Contrast}

Perceived contrast changes as a function of stimulus location within a given eccentricity (Fuller et al., 2008) and with eccentricity for a near threshold stimulus (Cannon, 1985). Could the higher perceived spatial frequency in both the simultaneous and delayed spatial frequency discrimination tasks along the horizontal meridian than along the vertical meridian be mediated by differences in perceived contrast? We think this was not the case, for the following reasons.

First, we used $100 \%$ contrast; both spatial frequency and orientation discrimination thresholds are independent 
of contrast at such high-contrast levels (Caelli, Brettel, Rentschler, \& Hilz, 1983; Regan, Bartol, Murray, \& Beverley, 1982; Regan \& Beverley, 1985; Skottun, Bradley, Sclar, Ohzawa, \& Freeman, 1987). In addition, when perceived contrast was measured by subjective contrastmatching between two high-contrast sinusoidal gratings, perceived contrast was constant for a wide range of spatial frequencies (Georgeson \& Sullivan, 1975).

Second, in experiments in which perceived spatial frequency was measured as a function of contrast, perceived spatial frequency increased as contrast decreased (Georgeson, 1985; Gobell \& Carrasco, 2005). Moreover, a low-contrast, transient stimulus looks finer (higher perceived frequency) than does a high-contrast, sustained stimulus (Gelb \& Wilson, 1983); had there been a shift in perceived spatial frequency due to a change in perceived contrast, therefore, it would have been in the opposite direction of the shift we found in Experiments 3 and 4 (see Figures 4A and 4D).

\section{CONCLUSION}

We set out to investigate (1) whether performance inhomogeneities emerge in spatial frequency discrimination, and (2) whether asymmetries present during encoding of visual information also emerge in VSTM tasks. This study revealed that spatial frequency discrimination and perceived spatial frequency, whether simultaneous or delayed, vary as a function of stimulus location. The HVA indicates that discrimination performance and perceived spatial frequency are higher along the horizontal than along the vertical meridian. This asymmetry in performance observed in perceptual discrimination tasks carried over to the delayed discrimination tasks engaging VSTM. These results indicate that factors that alter perceptual performance also affect memory performance. The quality with which a visual stimulus is perceived and encoded at different locations affects the quality with which that stimulus will be processed in VSTM.

\section{AUTHOR NOTE}

This research was supported by an NIH grant (RO1 EY016200) to M.C. We thank the members of M.C.'s lab and the three reviewers of the article for their valuable comments. Correspondence concerning this article should be addressed to L. Montaser-Kouhsari, Department of Psychology, New York University, 6 Washington Place, Room 957, New York, NY10003 (e-mail: 1mk306@nyu.edu).

\section{REFERENCES}

Berkley, M. A., Kitterle, F., \& Watkins, D. W. (1975). Grating visibility as a function of orientation and retinal eccentricity. Vision Research, 15, 239-244.

Bisley, J. W., \& PASTERnaK, T. (2000). The multiple roles of visual cortical areas MT/MST in remembering the direction of visual motion. Cerebral Cortex, 10, 1053-1065.

Brainard, D. H. (1997). The Psychophysics Toolbox. Spatial Vision, 10, 433-436.

Caelli, T., Brettel, H., Rentschler, I., \& Hilz, R. (1983). Discrimination thresholds in the two-dimensional spatial frequency domain. Vision Research, 23, 129-133.

Cameron, E. L., Tai, J. C., \& Carrasco, M. (2002). Covert attention affects the psychometric function of contrast sensitivity. Vision Research, 42, 949-967.
Cannon, M. W., JR. (1985). Perceived contrast in the fovea and periphery. Journal of the Optical Society of America A, 2, 1760-1768.

Carrasco, M., \& Chang, I. (1995). The interaction of objective and subjective organizations in a localization search task. Perception \& Psychophysics, 57, 1134-1150.

Carrasco, M., Evert, D. L., Chang, I., \& Katz, S. M. (1995). The eccentricity effect: Target eccentricity affects performance on conjunction searches. Perception \& Psychophysics, 57, 1241-1261.

Carrasco, M., Giordano, A. M., \& McElree, B. (2004). Temporal performance fields: Visual and attentional factors. Vision Research, 44, 1351-1365.

Carrasco, M., Talgar, C. P., \& Cameron, E. L. (2001). Characterizing visual performance fields: Effects of transient covert attention, spatial frequency, eccentricity, task and set size. Spatial Vision, 15, 61-75.

Carrasco, M., Williams, P. E., \& Yeshurun, Y. (2002). Covert attention increases spatial resolution with or without masks: Support for signal enhancement. Journal of Vision, 2, 467-479.

Cornelissen, F. W., \& Greenlee, M. W. (2000). Visual memory for random block patterns defined by luminance and color contrast. $V i$ sion Research, 40, 287-299.

Curcio, C. A., \& Allen, K. A. (1990). Topography of ganglion cells in human retina. Journal of Comparative Neurology, 300, 5-25.

Curcio, C. A., Sloan, K. R., Kalina, R. E., \& Hendrickson, A. E. (1990). Human photoreceptor topography. Journal of Comparative Neurology, 292, 497-523.

Deubel, H., \& SchneIDeR, W. X. (1996). Saccade target selection and object recognition: Evidence for a common attentional mechanism. Vision Research, 36, 1827-1837.

Edgar, G. K., \& Sмiтh, A. T. (1990). Hemifield differences in perceived spatial frequency. Perception, 19, 759-766.

FAHLE, M., \& HARRIS, J. P. (1992). Visual memory for vernier offsets. Vision Research, 32, 1033-1042.

Fuller, S., Rodriguez, R. Z., \& Carrasco, M. (2008). Apparent contrast differs across the vertical meridian: Visual and attentional factors. Journal of Vision, 8(1, Art. 16), 11-16.

Gelb, D. J., \& Wilson, H. R. (1983). Shifts in perceived size as a function of contrast and temporal modulation. Vision Research, 23, 71-82.

Georgeson, M. A. (1985). Apparent spatial frequency and contrast of gratings: Separate effects of contrast and duration. Vision Research, 25, 1721-1727.

Georgeson, M. A., \& Sullivan, G. D. (1975). Contrast constancy: Deblurring in human vision by spatial frequency channels. Journal of Physiology, 252, 627-656.

Gobell, J., \& Carrasco, M. (2005). Attention alters the appearance of spatial frequency and gap size. Psychological Science, 16, 644-651.

He, S., Cavanagh, P., \& Intriligator, J. (1996). Attentional resolution and the locus of visual awareness. Nature, 383, 334-337.

Hoffman, J. E., \& Subramaniam, B. (1995). The role of visual attention in saccadic eye movements. Perception \& Psychophysics, 57, 787-795.

Lalonde, J., \& Chaudhuri, A. (2002). Task-dependent transfer of perceptual to memory representations during delayed spatial frequency discrimination. Vision Research, 42, 1759-1769.

LEE, B., \& HARRIS, J. (1996). Contrast transfer characteristics of visual short-term memory. Vision Research, 36, 2159-2166.

Levine, M. W., \& MCANANY, J. J. (2005). The relative capabilities of the upper and lower visual hemifields. Vision Research, 45, 2820-2830.

Liu, T., Heeger, D. J., \& Carrasco, M. (2006). Neural correlates of the visual vertical meridian asymmetry. Journal of Vision, 6, 1294-1306.

Loftus, G. R., \& Masson, M. E. J. (1994). Using confidence intervals in within-subject designs. Psychonomic Bulletin \& Review, 1, 476-490.

Mackeben, M. (1999). Sustained focal attention and peripheral letter recognition. Spatial Vision, 12, 51-72.

Magnussen, S. (2000). Low-level memory processes in vision. Trends in Neurosciences, 23, 247-251.

Magnussen, S., \& Greenlee, M. W. (1992). Retention and disruption of motion information in visual short-term memory. Journal of Experimental Psychology: Learning, Memory, \& Cognition, 18, 151-156.

Magnussen, S., \& Greenlee, M. W. (1999). The psychophysics of perceptual memory. Psychological Research, 62, 81-92.

Magnussen, S., Greenlee, M. W., Asplund, R., \& Dyrnes, S. (1991). Stimulus-specific mechanisms of visual short-term memory. Vision Research, 31, 1213-1219. 
Magnussen, S., Greenlee, M. W., \& Thomas, J. P. (1996). Parallel processing in visual short-term memory. Journal of Experimental Psychology: Human Perception \& Performance, 22, 202-212.

Magnussen, S., IdÅs, E., \& Myhre, S. H. (1998). Representation of orientation and spatial frequency in perception and memory: A choice reaction-time analysis. Journal of Experimental Psychology: Human Perception \& Performance, 24, 707-718.

Mayfrank, L., Kimmig, H., \& Fischer, B. (1987). The role of attention in the preparation of visually guided saccadic eye movements in man. In J. K. O’ Regan \& A. Levy-Schoen (Eds.), Eye movements: From physiology to cognition (pp. 37-45). New York: North-Holland.

McAnany, J. J., \& Levine, M. W. (2007). Magnocellular and parvocellular visual pathway contributions to visual field anisotropies. Vision Research, 47, 2327-2336.

Nilsson, T. H., \& Nelson, T. M. (1981). Delayed monochromatic hue matches indicate characteristics of visual memory. Journal of Experimental Psychology: Human Perception \& Performance, 7, 141-150.

Pasternak, T., \& Greenlee, M. W. (2005). Working memory in primate sensory systems. Nature Reviews Neuroscience, 6, 97-107.

Pasternak, T., \& Zaksas, D. (2003). Stimulus specificity and temporal dynamics of working memory for visual motion. Journal of Neurophysiology, 90, 2757-2762.

Pelli, D. G. (1997). The VideoToolbox software for visual psychophysics: Transforming numbers into movies. Spatial Vision, 10, 437-442.

Perry, V. H., \& Cowey, A. (1985). The ganglion cell and cone distributions in the monkey's retina: Implications for central magnification factors. Vision Research, 25, 1795-1810.

REGAN, D. (1985). Storage of spatial-frequency information and spatialfrequency discrimination. Journal of the Optical Society of America A, 2, 619-621.

Regan, D., Bartol, S., Murray, T. J., \& Beverley, K. I. (1982). Spatial frequency discrimination in normal vision and in patients with multiple sclerosis. Brain, 105, 735-754.

Regan, D., \& Beverley, K. I. (1985). Postadaptation orientation discrimination. Journal of the Optical Society of America A, 2, 147-155.
RiJsdijK, J. P., Kroon, J. N., \& VAN der Wildt, G. J. (1980). Contrast sensitivity as a function of position on the retina. Vision Research, 20, 235-241.

Rovamo, J., \& Virsu, V. (1979). An estimation and application of the human cortical magnification factor. Experimental Brain Research, 37, 495-510.

Rovamo, J., Virsu, V., Laurinen, P., \& Hyvärinen, L. (1982). Resolution of gratings oriented along and across meridians in peripheral vision. Investigative Ophthalmology \& Visual Science, 23, 666-670.

Rubin, N., NAKAyama, K., \& Shapley, R. (1996). Enhanced perception of illusory contours in the lower versus upper visual hemifields, Science, 271, 651-653.

SAKAI, K. (2003). Short-term visual memory for contour curvature in a delayed discrimination task. Japanese Psychological Research, 45, 122-128.

Skottun, B. C., Bradley, A., Sclar, G., Ohzawa, I., \& Freeman, R. D. (1987). The effects of contrast on visual orientation and spatial frequency discrimination: A comparison of single cells and behavior. Journal of Neurophysiology, 57, 773-786.

TAlgar, C. P., \& CARrasco, M. (2002). Vertical meridian asymmetry in spatial resolution: Visual and attentional factors. Psychonomic Bulletin \& Review, 9, 714-722.

Vogels, R., \& Orban, G. A. (1986). Decision processes in visual discrimination of line orientation. Journal of Experimental Psychology: Human Perception \& Performance, 12, 115-132.

Wichmann, F. A., \& Hill, N. J. (2001a). The psychometric function: I. Fitting, sampling, and goodness of fit. Perception \& Psychophysics, 63, 1293-1313.

Wichmann, F. A., \& Hill, N. J. (2001b). The psychometric function: II. Bootstrap-based confidence intervals and sampling. Perception \& Psychophysics, 63, 1314-1329.

(Manuscript received December 28, 2008; revision accepted for publication May 28, 2009.) 